\title{
Electric and Magnetic Fields Generated by a Charged Bunch between Parallel Conducting Plates
}

\author{
B. Levchenko \\ Skobeltsyn Institute of Nuclear Physics, Lomonosov Moscow State University, 119991 Moscow, Russia \\ Correspondence should be addressed to B. Levchenko, levtchen@mail.desy.de \\ Received 30 September 2010; Accepted 8 November 2010 \\ Academic Editor: Han Pu
}

Copyright (c) 2010 B. Levchenko. This is an open access article distributed under the Creative Commons Attribution License, which permits unrestricted use, distribution, and reproduction in any medium, provided the original work is properly cited.

Image fields generated by a bunch of charged particles between two parallel perfectly conducting plates are studied in detail. We derive exact analytical expressions for external fields of a charged relativistic bunch with a circular cross-section. Summation of image fields by the direct method invented by Laslett allows the infinite series to be represented in terms of elementary trigonometric functions.

\section{Introduction}

In an accelerator, the charged beam is influenced by an environment (a beam pipe, accelerator gaps, magnets, collimators, etc.), and a high-intensity bunch induces surface charges or currents into this environment. This modifies the electric and magnetic fields around the bunch. There is a relatively simple method to account for the effect of the environment by introducing image charges and currents.

Methods of image fields summation are described in the paper [1], which presents some field coefficients calculated for infinite parallel-plate vacuum chambers, magnetic poles, and vacuum chambers with elliptical cross-sections and variable aspect rations. The resulting image field was calculated only in the linear approximation and depends linearly on the deviations $\bar{x}$ and $x$ of the bunch center and the position of a test particle, respectively, from the axis. The approximation used is incorrect if the field observation point $x$ is located far from the bunch or if the bunch center is close to a conducting wall.

In the present paper we consider the classical problem of the image field summation once again for a very simple geometry, namely, a relativistic bunch moving between infinitely wide parallel perfectly conducting planes. The problem is far from being a pure academic one $[2,3]$. In applications, in particular by study of the electron cloud effect [4] and the dynamics of photoelectrons in the beam transport system, it is important to know the distribution of electromagnetic fields not only in vicinity of the bunch but also in the whole collimator gap.

In free space, the external radial electric $\vec{E}_{\perp}$ and azimuthal magnetic $\vec{B}_{\phi}$ self-fields of a cylindrical bunch with a uniform charge density are described by [5-7]

$$
\begin{gathered}
E_{\perp}(r)=\kappa \frac{2 \lambda}{r}, \\
B_{\phi}=-\frac{\beta}{c} E_{\perp}(r),
\end{gathered}
$$

where $\kappa=1 / 4 \pi \epsilon_{0}, \lambda$ is the linear charge density, $\beta=v / c$ the normalized velocity of the beam constituents, and $c$ the velocity of light.

We did not find publications with attempts to sum up the series (3) in an approximation beyond the linear one. In the following sections we present the exact solutions of the problem.

\section{Electric Field from Image Charges}

Following Laslett [1] (see also [5]), we consider a relativistic bunch of the length $L$ between infinitely wide conducting plates at $x= \pm h$. Suppose that constituents of the bunch are positively charged. For full generality, let the circular particle bunch be displaced in the horizontal plane by $\bar{x}$ from the midplane $(0, y, z)$, and the observation point of the field be at $(x, 0,0)$ between the conducting parallel plates. The end 
points of the bunch are at $z= \pm L / 2$. The boundary condition for electric fields is $E_{z}( \pm h)=0$ on the conducting plane and is satisfied if the image charges change sign from image to image. Suppose that the distance between plates is of the order $L$. Thus, the electric field of each image is described by (1). To calculate the image electric field $E_{\perp \text {,image }}(x)$ in front of the plate, we add the contributions from all image fields in the infinite series [1] (see also $[5,8]$ )

$$
\begin{aligned}
& E_{\perp, \text { image }}(x, \bar{x}) \\
& =2 \kappa \lambda \cdot\left\{\left(2 h-x_{1}\right)^{-1}-\left(2 h+x_{1}\right)^{-1}-\left(4 h-x_{2}\right)^{-1}\right. \\
& +\left(4 h+x_{2}\right)^{-1}+\left(6 h-x_{1}\right)^{-1}-\left(6 h+x_{1}\right)^{-1} \\
& \quad-\left(8 h-x_{2}\right)^{-1}+\left(8 h+x_{2}\right)^{-1} \\
& +\left(10 h-x_{1}\right)^{-1}-\left(10 h+x_{1}\right)^{-1} \\
& \left.-\left(12 h-x_{2}\right)^{-1}+\left(12 h+x_{2}\right)^{-1}+\cdots\right\},
\end{aligned}
$$

where $x_{1}=x+\bar{x}$ and $x_{2}=x-\bar{x}, \lambda=q N / L$ and $N$ is the number of particles of charge $q$ per bunch. These image fields must be added to the direct field of the bunch (1) to meet the boundary condition that the electric field enters conducting surfaces perpendicularly.

In the original paper [1], the series (3) was summed up only in the linear approximation in $x$ and $\bar{x}$,

$$
E_{\perp, \text { image }}(x, \bar{x})=4 \kappa \lambda \frac{\epsilon_{1}}{h^{2}}(2 \bar{x}+x)
$$

The coefficient $\epsilon_{1}=\pi^{2} / 48$ is known as the Laslett coefficient (or form factor) for infinite parallel-plate vacuum chambers and magnetic poles. The approximation (4) widespread in textbooks and lectures is however incorrect if the deviation of the bunch center from the axis is large $(\bar{x} \sim h)$ or if the field observation point $x$ is located far off the bunch. Therefore, below we present the exact solution of the problem.

In the appendix we prove that the exact summation of the series (3) gives

$$
E_{\perp, \text { image }}(x, \bar{x})=\frac{4 \kappa \lambda}{h} \Lambda(\delta, \bar{\delta}),
$$

where the image field structure function $\Lambda$ depends only on normalized variables $\delta=x / h, \bar{\delta}=\bar{x} / h$ in the form

$$
\Lambda(\delta, \bar{\delta})=\frac{1}{2}\left[\frac{\pi}{2} \cdot \frac{\cos ((\pi / 2) \bar{\delta})}{\sin ((\pi / 2) \delta)-\sin ((\pi / 2) \bar{\delta})}-\frac{1}{\delta-\bar{\delta}}\right] .
$$

In the appendix it is also shown that in the linear approximation equation (5) recovers the part (4) derived by Laslett.

We will now estimate values of the function $\Lambda$ in several particular field points. If the observation point of the field is located at the plate, $x=h$, then $\delta=1$ and the structure function depends only on the bunch center position between plates, $\bar{\delta}$. Thus, from (6) one gets

$$
\Lambda(1, \bar{\delta})=\frac{1}{2}\left\{\frac{\pi}{2} \frac{1+\sin ((\pi / 2) \bar{\delta})}{\cos ((\pi / 2) \bar{\delta})}-\frac{1}{1-\bar{\delta}}\right\} .
$$

Equation (7) is singular at $\bar{\delta} \rightarrow 1$ and shows that the conducting plate attracts the bunch with increasing force with the bunch displacement from the midplane. This phenomenon, involving the transverse movement of the bunch as a whole, arises from image forces and could lead to a transverse instability of the bunch.

For the bunch in the midplane, $\bar{\delta}=0$, the summed image field at the surface equals

$$
E_{\perp, \text { image }}(h, 0)=\frac{4 \kappa \lambda}{h} \Lambda(1,0)=\frac{2 \kappa \lambda}{h}\left(\frac{\pi}{2}-1\right) .
$$

The image field (5) must be added to the direct field of the bunch (1) to meet the boundary condition. Thus

$$
\begin{aligned}
E_{\perp, \mathrm{tot}}(x, \bar{x}) & =E_{\perp, \mathrm{dir}}+E_{\perp, \text { image }} \\
& =\frac{\pi \kappa \lambda}{h} \cdot \frac{\cos ((\pi / 2) \bar{\delta})}{\sin ((\pi / 2) \delta)-\sin ((\pi / 2) \bar{\delta})} .
\end{aligned}
$$

For a bunch in the midplane, $\bar{\delta}=0$, we find from (9) the expression of the transverse component of electric field generated by a relativistic bunch moving between wide conducting parallel plates

$$
E_{\perp, \mathrm{tot}}(x, 0)=\frac{2 \kappa \lambda}{x} \cdot \frac{(\pi / 2) \delta}{\sin ((\pi / 2) \delta)} .
$$

That is, at the surface, $\delta=1$, the field is enhanced by a factor $\pi / 2$ due to the presence of the conducting plates.

Notice that in the linear approximation (4) the field gradient, $\partial E_{\perp, \mathrm{im}} / \partial x$, is independent of position $x$. Thus the tune shift experienced by each particle in the bunch is the same (a coherent tune shift). However, the exact result (5) demonstrates that the coherence is violated and (5) allows to estimate the accuracy of the linear approximation.

\section{Magnetic Images}

In the above, we have used electrostatic images. Magnetic images can be treated in much the same way. Let the ferromagnetic boundaries be represented by a pair of infinitely wide parallel surfaces at $x= \pm g$. The magnetic field lines must enter the magnetic pole faces perpendicularly. For magnetic image fields we distinguish between DC and AC image fields. The DC field penetrates the metallic vacuum chamber and reaches the ferromagnetic poles. In case of bunched beams the AC fields are of rather high frequency and we assume that they do not penetrate the thick metallic vacuum chamber.

A magnetic field seen by a particle at location $x$ on the $x$-axis is generated by the successive image currents with the 
same sign as the beam itself $[7,8]$. Therefore, instead of the series (A.1) we get

$$
\begin{aligned}
(2 g- & \left.x_{1}\right)^{-1}-\left(2 g+x_{1}\right)^{-1}+\left(4 g-x_{2}\right)^{-1} \\
& -\left(4 g+x_{2}\right)^{-1}+\left(6 g-x_{1}\right)^{-1}-\left(6 g+x_{1}\right)^{-1} \\
& +\left(8 g-x_{2}\right)^{-1}-\left(8 g+x_{2}\right)^{-1}+\left(10 g-x_{1}\right)^{-1} \\
& \quad-\left(10 g+x_{1}\right)^{-1}+\left(12 g-x_{2}\right)^{-1} \\
& -\left(12 g+x_{2}\right)^{-1}+\cdots \\
= & \sum_{k}^{\infty} \Pi_{k}\left(x_{1}, g\right)+\sum_{m}^{\infty} \Pi_{m}\left(x_{2}, g\right)=\frac{2}{g} H\left(\eta_{1}, \eta_{2}\right),
\end{aligned}
$$

where $\Pi_{k}$ and $\Pi_{m}$ are of the same functional form as (A.3) with an interchange of variables $h \rightarrow g, \delta_{1} \rightarrow \eta_{1}=x_{1} / g$, and $\delta_{2} \rightarrow \eta_{2}=x_{2} / g$. Now, if to proceed in the same manner as in the appendix, we obtain from (11) an expression for the structure function of the image magnetic field

$$
H(\eta, \bar{\eta})=\frac{1}{2}\left[\frac{1}{\eta-\bar{\eta}}-\frac{\pi}{2} \cdot \frac{\cos ((\pi / 2) \eta)}{\sin ((\pi / 2) \eta)-\sin ((\pi / 2) \bar{\eta})}\right] .
$$

Thus, the resulting DC field is described by

$$
B_{y, \text { image, DC }}(x, \bar{x})=\frac{4 \kappa \lambda \beta}{c g} \cdot \mathcal{B} \cdot H(\eta, \bar{\eta}) \text {, }
$$

where $\eta=x / g$ and $\bar{\eta}=\bar{x} / g, \mathcal{B}=n_{b} L / 2 \pi R$ is the bunching factor, $n_{b}$ the number of bunches, and $R$ the average accelerator radius.

The contribution of magnetic AC image field due to eddy currents in vacuum chamber walls is similar to electric image fields

$$
B_{y \text {,image, AC }}(x, \bar{x})=-\frac{4 \kappa \lambda \beta}{c h} \cdot(1-\mathscr{B}) \cdot \Lambda(\delta, \bar{\delta}),
$$

where the factor $(1-\mathscr{B})$ accounts for the subtraction of the DC component.

The magnetic image fields must be added to the direct magnetic field (2) from the bunch to meet the boundary condition of normal components at ferromagnetic surfaces. That is, the total magnetic field between the conducting plates is

$$
\begin{aligned}
& B_{y, \text { tot }}(x, \bar{x})= B_{y}+B_{y, \text { image, DC }}+B_{y, \text { image, AC }} \\
&=-\frac{\pi \kappa \lambda \beta}{c h}\left\{\frac{(1-\mathcal{B}) \cos ((\pi / 2) \bar{\delta})}{\sin ((\pi / 2) \delta)-\sin ((\pi / 2) \bar{\delta})}\right. \\
&\left.+\frac{h}{g} \cdot \frac{\mathscr{B} \cos ((\pi / 2) \eta)}{\sin ((\pi / 2) \eta)-\sin ((\pi / 2) \bar{\eta})}\right\} .
\end{aligned}
$$

\section{Summary}

We reanalyzed the classical problem of summing image fields generated by a bunch of charged particles moving with a relativistic velocity between infinitely wide parallel perfectly conducting plates. The exact solutions of the problems are represented by (6) and (13) for the structure function $\Lambda(\delta, \bar{\delta})$ of electric images and the structure function $H(\eta, \bar{\eta})$ of magnetic images, depending only on the normalized variables.

\section{Appendix}

Here we derive the main formula (6).

Let us split the contribution of all image fields (3) given in braces into two parts,

$$
\begin{aligned}
(2 h- & \left.x_{1}\right)^{-1}-\left(2 h+x_{1}\right)^{-1}-\left(4 h-x_{2}\right)^{-1} \\
& +\left(4 h+x_{2}\right)^{-1}+\left(6 h-x_{1}\right)^{-1}-\left(6 h+x_{1}\right)^{-1} \\
& -\left(8 h-x_{2}\right)^{-1}+\left(8 h+x_{2}\right)^{-1}+\left(10 h-x_{1}\right)^{-1} \\
& -\left(10 h+x_{1}\right)^{-1}-\left(12 h-x_{2}\right)^{-1} \\
& +\left(12 h+x_{2}\right)^{-1}+\cdots \\
= & \sum_{k}^{\infty} \Pi_{k}\left(x_{1}, h\right)-\sum_{m}^{\infty} \Pi_{m}\left(x_{2}, h\right),
\end{aligned}
$$

where $\Pi_{k}$ represents the contribution from the negativecharged images and $\Pi_{m}$ is the contribution from the positivecharged images. Here and hereinafter, indexes $k$ and $m$ possess odd, $k=1,3,5, \ldots$, and even, $m=2,4,6, \ldots$, values.

An expansion of denominators of $\Pi_{k}$ and $\Pi_{m}$ into a power series of small parameters $\delta_{1}=x_{1} / h<1$ and $\delta_{2}=$ $x_{2} / h<1$ gives

$$
\begin{aligned}
\Pi_{k}\left(x_{1}, h\right) & =\frac{1}{2 k h-x_{1}}-\frac{1}{2 k h+x_{1}} \\
& =\frac{2 x_{1}}{(2 k h)^{2}-x_{1}^{2}}=\frac{2}{h} \sum_{n=1}^{\infty} \frac{\delta_{1}^{2 n-1}}{(2 k)^{2 n}}, \\
\Pi_{m}\left(x_{2}, h\right) & =\frac{1}{2 m h-x_{2}}-\frac{1}{2 m h+x_{2}} \\
& =\frac{2 x_{2}}{(2 m h)^{2}-x_{2}^{2}}=\frac{2}{h} \sum_{n=1}^{\infty} \frac{\delta_{2}^{2 n-1}}{(2 m)^{2 n}} .
\end{aligned}
$$

Now it is evident that the space structure of the image fields between plates is characterized by a specific function $\Lambda\left(\delta_{1}, \delta_{2}\right)$, we term it the structure function,

$$
\sum_{k}^{\infty} \Pi_{k}-\sum_{m}^{\infty} \Pi_{m}=\frac{2}{h} \Lambda\left(\delta_{1}, \delta_{2}\right)
$$


with

$$
\begin{aligned}
\Lambda\left(\delta_{1}, \delta_{2}\right)= & \sum_{k}^{\infty}\left[\frac{\delta_{1}}{(2 k)^{2}}+\frac{\delta_{1}^{3}}{(2 k)^{4}}+\cdots\right] \\
& -\sum_{m}^{\infty}\left[\frac{\delta_{2}}{(2 m)^{2}}+\frac{\delta_{2}^{3}}{(2 m)^{4}}+\cdots\right] .
\end{aligned}
$$

The structure function $\Lambda$ depends only on the normalized variables.

To proceed further, let us define the following auxiliary quantities:

$$
\begin{aligned}
M_{j}^{(-)} & =\sum_{k}^{\infty} \frac{1}{(2 k)^{2 j}}-\sum_{m}^{\infty} \frac{1}{(2 m)^{2 j}}=\sum_{n=1}^{\infty} \frac{(-1)^{n+1}}{(2 n)^{2 j}} \\
& =\frac{1}{2^{2 j}} \cdot \frac{\left(2^{2 j-1}-1\right) \pi^{2 j}}{(2 j) !}\left|B_{2 j}\right|, \\
M_{j}^{(+)} & =\sum_{k}^{\infty} \frac{1}{(2 k)^{2 j}}+\sum_{m}^{\infty} \frac{1}{(2 m)^{2 j}}=\sum_{n=1}^{\infty} \frac{1}{(2 n)^{2 j}} \\
& =\frac{1}{2^{2 j}} \cdot \frac{2^{2 j-1} \cdot \pi^{2 j}}{(2 j) !}\left|B_{2 j}\right|,
\end{aligned}
$$

where $B_{2}$ are Bernoulli numbers, $B_{2}=1 / 6, B_{4}=-1 / 30$, $B_{6}=1 / 42$, and so forth. By adding and subtracting the leftmost parts of (A.6), we express $k$ and $m$ numerical series of (A.5) in terms of $M_{j}^{(-)}$and $M_{j}^{(+)}$. Therefore, we get from (A.5)

$$
\begin{aligned}
& \Lambda\left(\delta_{1}, \delta_{2}\right) \\
& =\frac{1}{2} \sum_{n=1}^{\infty}\left[\left(M_{n}^{(-)}+M_{n}^{(+)}\right) \delta_{1}^{2 n-1}+\left(M_{n}^{(-)}-M_{n}^{(+)}\right) \delta_{2}^{2 n-1}\right],
\end{aligned}
$$

or after substituting (A.6) in (A.7), we find the following form of the structure function generated by the charged bunch:

$$
\Lambda\left(\delta_{1}, \delta_{2}\right)=\frac{1}{2} \sum_{n=1}^{\infty}\left[\left(2^{2 n}-1\right) \delta_{1}^{2 n-1}-\delta_{2}^{2 n-1}\right] \frac{\pi^{2 n}}{2^{2 n}(2 n) !}\left|B_{2 n}\right| .
$$

Using only the linear terms we recover the part derived by Laslett [1] (see (4))

$$
\Lambda(\bar{x}, x, h)=\frac{1}{h} \cdot \epsilon_{1}(2 \bar{x}+x) .
$$

An inspection of (A.8) shows that the contributions of negative-charged images are enhanced by the factor $2^{2 n}-1$, as compared with the contributions from the positive-charged images. Equation (A.8) also shows that for $x$ in the bunch center, $\delta_{2}=0$ and the contributions from the positivecharged images vanish.

At the final step, it is possible to rewrite the infinite series (A.8) in terms of elementary trigonometric functions. To do this, recall the relations between the Bernoulli numbers and the trigonometric functions [9-11]

$$
\begin{gathered}
z \tan (z)=\sum_{n=1}^{\infty} \frac{\left(2^{2 n}-1\right)(2 z)^{2 n}}{(2 n) !}\left|B_{2 n}\right|, \\
z \cot (z)=1-\sum_{n=1}^{\infty} \frac{(2 z)^{2 n}}{(2 n) !}\left|B_{2 n}\right| .
\end{gathered}
$$

After some algebraic manipulations and the use of (A.10), we get from (A.8) a new exact and compact expression of the structure function

$$
\Lambda\left(\delta_{1}, \delta_{2}\right)=\frac{1}{2}\left[\frac{\pi}{4} \tan \left(\frac{\pi}{4} \delta_{1}\right)+\frac{\pi}{4} \cot \left(\frac{\pi}{4} \delta_{2}\right)-\frac{1}{\delta_{2}}\right] .
$$

Now, if we recall that $\delta_{1}=(x+\bar{x}) / h=\delta+\bar{\delta}$ and $\delta_{2}=(x-$ $\bar{x}) / h=\delta-\bar{\delta}$, we obtain

$$
\Lambda(\delta, \bar{\delta})=\frac{1}{2}\left[\frac{\pi}{2} \cdot \frac{\cos ((\pi / 2) \bar{\delta})}{\sin ((\pi / 2) \delta)-\sin ((\pi / 2) \bar{\delta})}-\frac{1}{\delta-\bar{\delta}}\right] .
$$

At a first glance, (A.11) or (A.12) is singular at $\delta_{2}=0$ or $\delta=\bar{\delta}$, respectively. However, as we already discussed right after (A.8), this is not the case. Starting once again from (A.8) with $\delta_{2}=0$ and accounting (A.10), we get formally

$$
\Lambda(\bar{\delta}, \bar{\delta})=\frac{\pi}{8} \tan \left(\frac{\pi}{2} \bar{\delta}\right)
$$

\section{Acknowledgments}

The author is grateful to P. Bussey, E. Lohrmann, M. Dohlus, and F. Willeke for reading the draft paper, comments and useful discussions. This study is partially supported by the Russian Foundation for Basic Research under Grant no. 0502-39028.

\section{References}

[1] L. J. Laslett, "On intensity limitations imposed by transverse space-charge effects in circular particle accelerators," in Proceedings of the BNL Summer Study on Storage Rings, BNL-7534, pp. 324-367, 1963.

[2] B. B. Levchenko, "On field emission in high energy colliders initiated by a relativistic positively charged bunch of particles," http://arxiv.org/abs/physics/0608135.

[3] B. B. Levchenko, "Real and image fields of a relativistic bunch," http://arxiv.org/abs/physics/0604013.

[4] R. Cimino, I. R. Collins, M. A. Furman et al., "Can low-energy electrons affect high-energy physics accelerators?" Physical Review Letters, vol. 93, no. 1, Article ID 014801, 2004.

[5] H. Wiedemann, Particle Accelerator Physics: Vol. 2: Nonlinear and Higher Order Beam Dynamics, Springer, Berlin, Germany, 1995.

[6] M. Sands, "The physics of electron storage rings: an introduction," SLAC-0121, 1970.

[7] A. W. Chao, Physics of Collective Beam Instabilities in HighEnergy Accelerators, Wiley, New York, NY, USA, 1993. 
[8] A. Hofmann, In Jyvaeskylae 1992, Proceedings, General accelerator physics, vol. 1. CERN Geneva-CERN-94-01 (94/01,rec.Mar.) pp. 329-348, 1994.

[9] I. S. Gradshteyn and I. M. Ryzhik, Table of Integrals, Series, and Products, Nauka, Moscow, Russia, 1971, Academic Press, New York, NY, USA, 1980.

[10] L. Cong, "On Bernoulli Numbers and Its Properties," 2004, http://www.citebase.org/abstract?id=oai:arXiv.org:math 10408082.

[11] T. Kim, "Euler numbers and polynomials associated with zeta functions," Abstract and Applied Analysis, vol. 2008, Article ID 581582, 11 pages, 2008. 

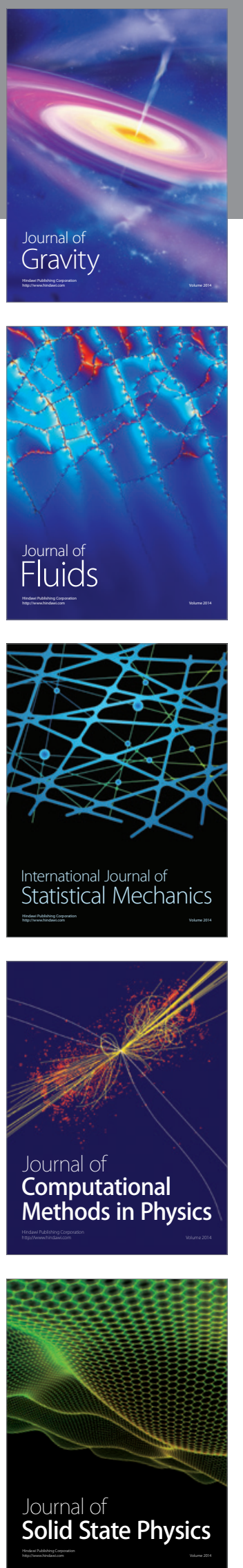

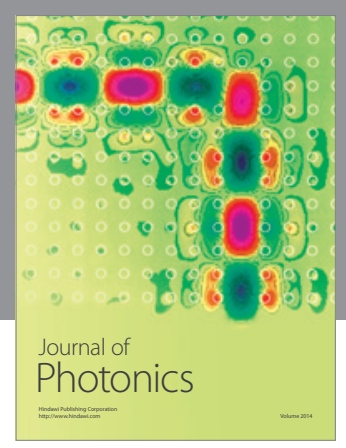

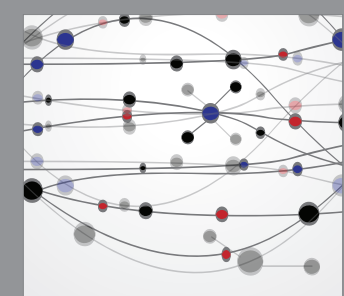

The Scientific World Journal
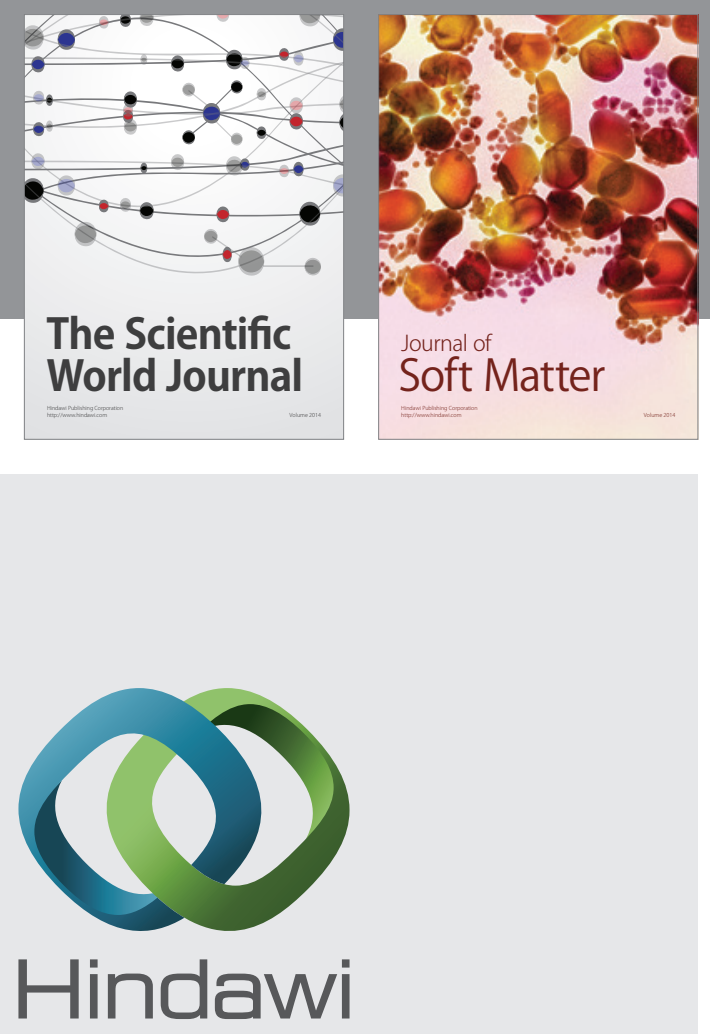

Submit your manuscripts at

http://www.hindawi.com
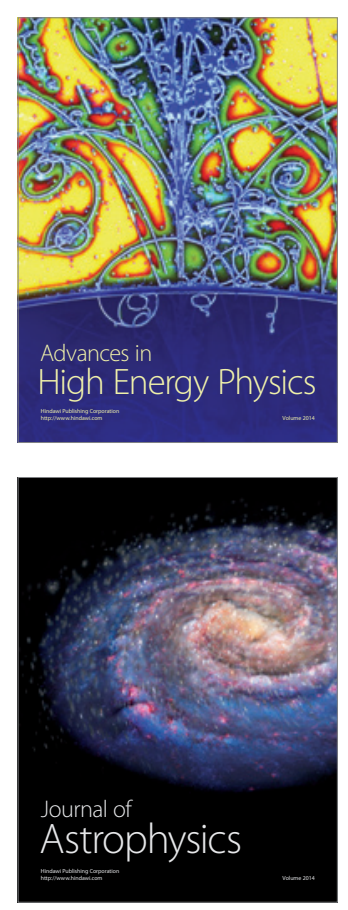
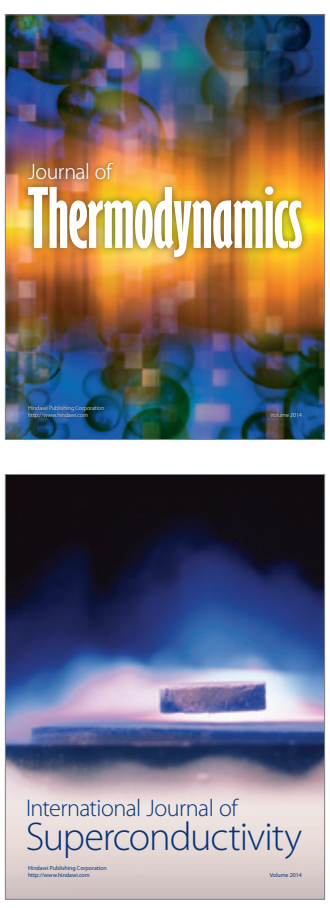
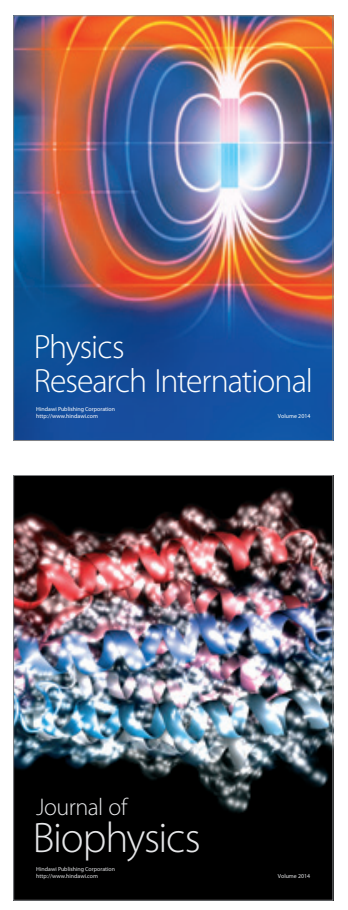
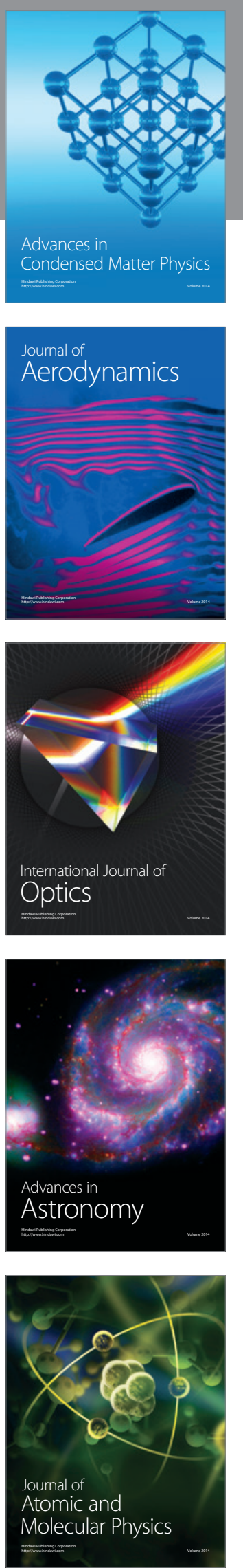